\title{
Scaling Study of Pseudospark Produced Electron Beam*
}

\author{
K. K. Jain, ${ }^{\dagger}$ B. N. Ding, ${ }^{\ddagger}$ and M. J. Rhee \\ Laboratory for Plasma Research and \\ Electrical Engineering Department \\ University of Maryland \\ College Park, Maryland 20742
}

\section{Abstract}

The characteristics of the breakdown voltage of a pseudospark device, the current, and the rms emittance of clectron beam produced in the device are determined. It is found that the breakdown voltage is a function of the product of the gas pressure squared and the anode-cathode gap distance; the electron beam current increases with the breakdown voltage up to $35 \mathrm{kV}$ and then decreases; the rus emittance appears to be nearly constant up to the breakdown voltage $25 \mathrm{kV}$ and then increases.

\section{INTRODUCTION}

Recently, the pscudospark discharge[1-8] has gained considerable attention because of its interesting discharge characteristic[2-4] as well as its capability of producing a high-quality electron beam.[5-8] It is imperative to determine the characteristics of the breakdown voltage, the clectron beam current, and the beam quality of the pseudospark device not only for a better understanding of the discharge but also for the scaling of further applications.

In this work, we report least-squares-fit analyses of breakdown voltages of a multigap pseudospark device, pesak currents and rms emittances of the electron beam produced in the device. The results show that the breakdown voltage is a function of $p^{2} d$; the electron beam current increases up to $35 \mathrm{kV}$ and then decreases and it increases linearly with the capacitance; the rms emittance appears to be nearly constant up to $25 \mathrm{kV}$ and then increases.

\section{EXPERIMENT}

The experimental setup is shown in Fig. 1. The discharge chamber consists of a planar cathode with a hollow carity, a number of sets of intermediate electrodes and insulators, and a planar anode. A center hole is present through the entire electrode system. The anode-cathode gap distance is varied by employing different number of intermediate electrode sets (each set is $6.4 \mathrm{~mm}$ thick). The

- This work was supported by the U.S. Department of Energy.

†Present address: Institute for Plasma Research, Bhat, Gindhinagar-382 424, India

Permanent address: China Academy of Engineering Physics, P.O. Box 523-56, Chengdy, Sichuan, China

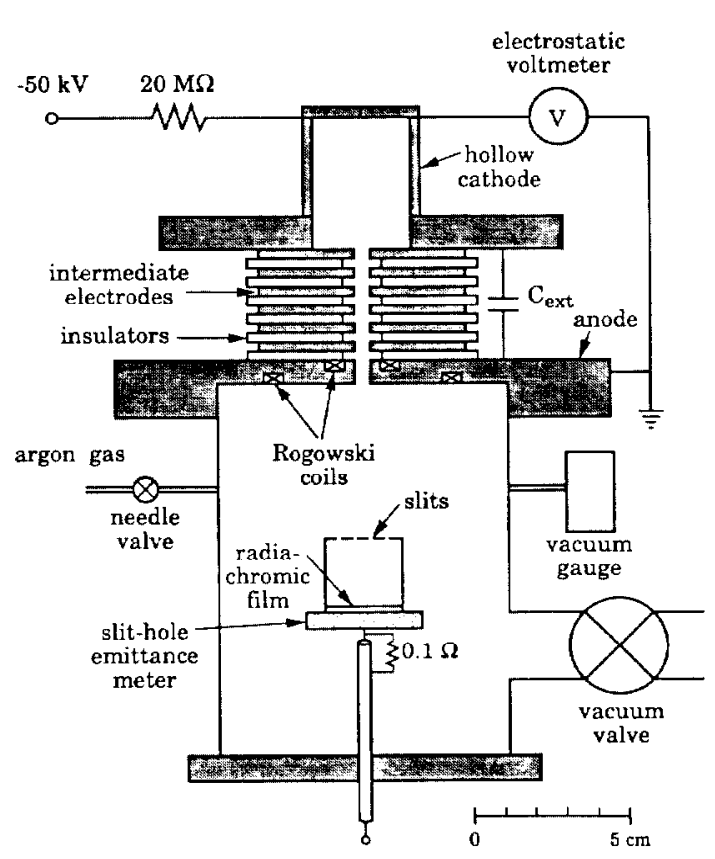

Figure 1: Experimental setup.

capacitance as initial energy storage of the chamber is varied by connecting different combination of low inductance type capacitors. Argon gas is used in this experiment and the pressure is measured by a capacitance-manometer type vacuum gauge. The cathode side of the chamber is charged up to $-50 \mathrm{kV}$ through a $20 \mathrm{M} \Omega$ charging resistor. The charging voltage is measured by an electrostatic voltmeter. The electron beam current is measured by a Rogowski coil and a Faraday cup. The emittance meter consists of a series of parallel thin slits of $200 \mu \mathrm{m}$ width and $2 \mathrm{~mm}$ spacing constructed from $0.5 \mathrm{~mm}$ stainless steel plate and $2 \mathrm{mil}$ thick radiachromic film used as a bcam detcctor, which is placed $10 \mathrm{~mm}$ downstream of the slit plane.

Initially the chamber is evacuated by an oil diffusion pump typically down to $10^{-5}$ Torr. The cathode side of the chamber is then charged to a given voltage. The argon gas pressure of the chamber is then increased at a very slow rate, $d p / d t \approx 1 \mathrm{mTor} / \mathrm{sec}$, until breakdown takes place. For the given voltage, the pressures at which the break- 
down takes place and the waveforms of the electron beam current produced are recorded for a wide range of experimental parameters: voltage, anode-cathode gap distance, and capacitance.

\section{The Breakdown Voltage}

In order to determine the functional dependence, the measured pressures are lcast-squares fitted to a regression plane given by a simple two variable function

$$
p(d, v)=a v^{b} d^{c}
$$

where $p, v$, and $d$ are the pressure in Torr, voltage in $\mathrm{kV}$, and the anode-cathode gap distance in mm, respectively, and the coefficients $a, b$, and $c$ are to be determined.

The least-squares fitting is done by employing a numerical method, called the grid search, described in Ref. 9. The algorithm of the method includes the following steps: Starting with the initial values of the coefficients $a, b$, and $c$, chi-square is evaluated as $\left.\chi^{2}=\sum\left[p_{i}-a v_{i}^{b} d_{i}^{c}\right)\right]^{2} /(N-$ $n-1)$ for the entire data set, where $N=250$ is the total number of data points and $n=3$ is the number of coefficients. The value of a is then increased (or decreased) by a small amount and $\chi^{2}$ is again evaluated to compare with the previous value. This step is repeated until $\chi^{2}$ reaches its minimum. The same procedure is repeated for the coefficients $b$ and $c$. All of the above steps are iterated many times until $\chi^{2}$ converges to a minimum value at which point the values of $a, b$, and $c$ for the best fit are obtained.

The results of this numerical analysis are summarized in the following: $a \pm \sigma_{a}=0.6851 \pm 0.0003, b \pm \sigma_{b}=$ $-0.2252 \pm 0.0014, c \pm \sigma_{c}=-0.5012 \pm 0.0016$. The regression plane with the least-squares-fit coefficients is depicted by the solid lines and plotted together with the data points in Fig. 2. It should be noted that the choice of $p$ as the dependent variable in $\mathrm{Eq}$. (1) instead of $v$ (or $d$ ) is because of the convergence speed in the numerical calculation. Equation (1) is rewritten in a more traditional form,

$$
v(p, d)=\alpha /\left(p^{\beta} d\right)^{\delta}
$$

where $\alpha=0.1865 \pm 0.0019, \beta=1.9952 \pm 0.0064$, and $\delta=2.226 \pm 0.016$ are found from the values of coefticients $a, b$, and $c$. It is interesting to note that the value of $\beta$ is very close to 2 which is well within the error range. This suggests that the breakdown voltage is a function only of the product, $p^{2} d$. Other results[10] show a dependence of $p d^{0}{ }^{58}$, which seems to closely support this $p^{2} d$ dependence.

\section{The Electron Beam Current}

The peak current of the electron beam measured with the anode-cathode gap distance of $38.4 \mathrm{~mm}$ is least-squares fitted to a function

$$
I_{p}=\left(a_{1}+a_{2} C\right) \exp \left[-\left(V-a_{3}\right)^{2} / 2 a_{4}^{2}\right],
$$

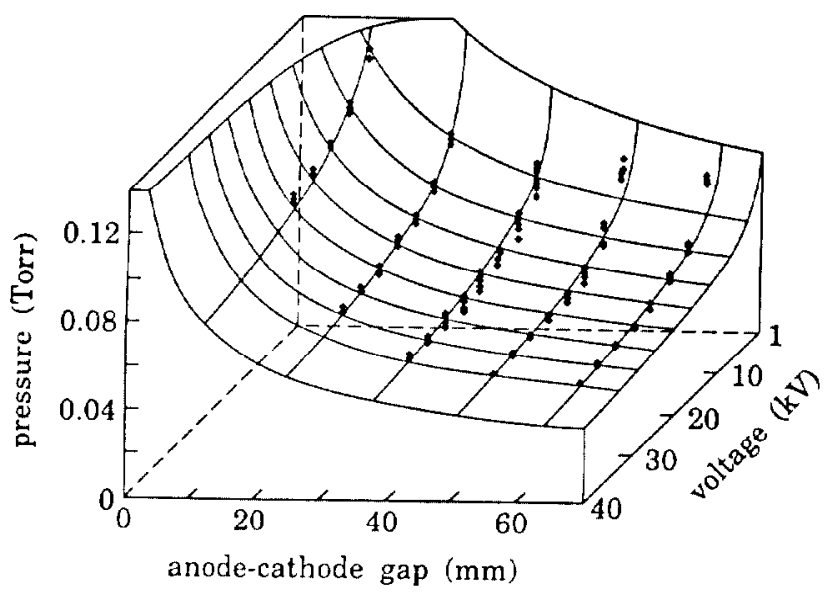

Figure 2: Measured pressures and the least-squares-fit regression plane.

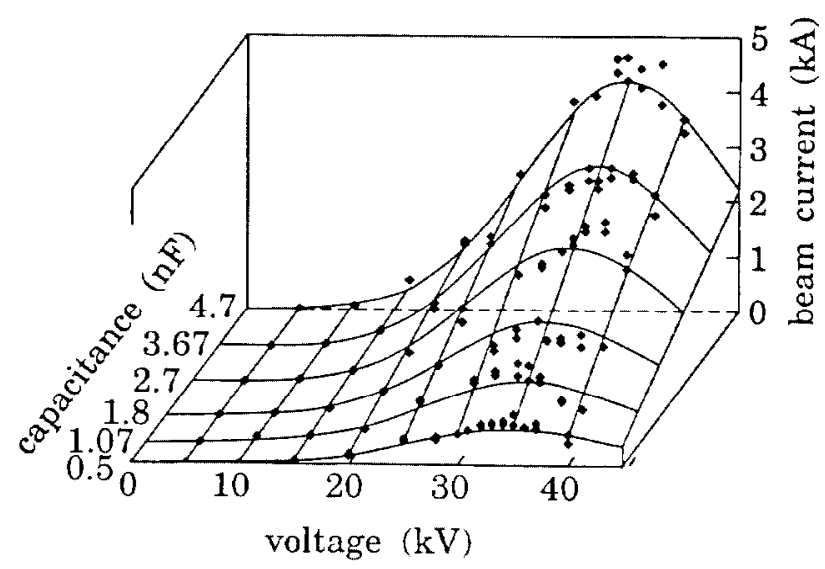

Figure 3: Measured peak currents and the least-squares-fit regression plane.

where $C$ is the storage capacitance $(\mathrm{nF}), V$ is the breakdown voltage $(\mathrm{kV}), a_{1}, a_{2}, a_{3}$, and $a_{4}$ are the coefficients to be determined. The same numerical method described above is used. The coefficients for the best fit are found to be $a_{1}=213.6(\mathrm{~A}), a_{2}=842.4(\mathrm{~A} / \mathrm{nF}), a_{3}=34.76(\mathrm{kV})$ and $a_{4}=9.05(\mathrm{kV})$. The resultant regression plane and data points are shown in Fig. 3.

\section{The RMS Emittance}

For the emittance measurement, exposure of approximately ten consecutive beam pulses to a radiachromic film through the slits in the emittance meter is made. An attempt is made to scale the emittance with the breakdown voltage by measuring the emittance at several different breakdown voltages. Typical optical density profile of the radiachromic film after exposure is shown in Fig. 4. It is very reasonable to assume that as in Ref. 11 , the beam produced in this experiment is axisymmetric and of Maxwellian transverse velocity distribution. This allows us to use the simple slit-hole type emittance meter, whose results can be easily analyzed.[11] The emittances vs the breakdown voltages are plotted in Fig. 5 . 


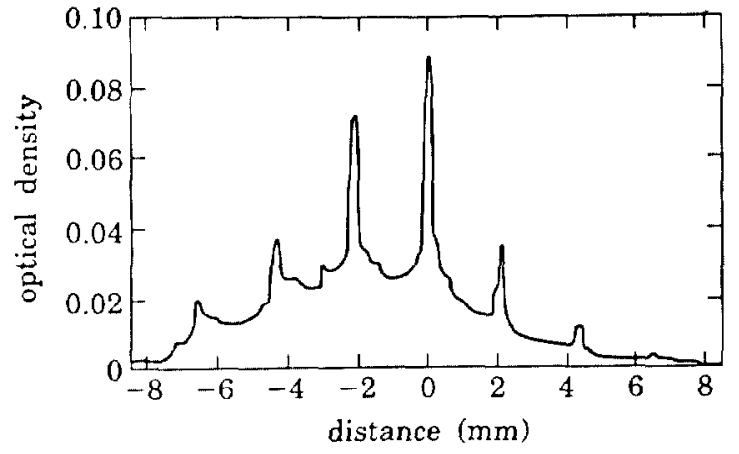

Figure 4: Typical optical density profile of the radiachromic film.

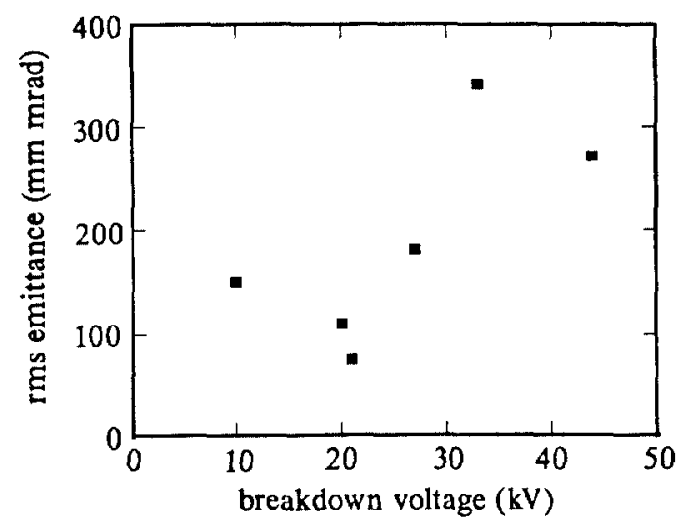

Figure 5: The rms emittance vs the breakdown voltage.

\section{Conclusions}

In conclusion, the breakdown voltage of a pseudospark device, the peak current and rms emittance of the electron beam produced in the device have been measured for a wide range of gas pressure, anode-cathode gap distance, and storage capacitance. The measured data are analyzed by a least-squares-fit method. It is found that the breakdown voltage is a function of the product of the gas pressure squared and the anode-cathode gap distance; the electron beam current increases linearly with the capacitance and increases with the breakdown voltage up to $35 \mathrm{kV}$ and then decreases; the the rms emittance is nearly constant up to $25 \mathrm{kV}$ and then increases.

\section{REFERENCES}

[1] J. Christiansen and C. Schultheiss, Z. Physik A 290, 35 (1979).

[2] D. Bloess, I. Kamber, H. Riege, G. Bittner, V. Bruckner, J. Christiansen, K. Frank, W. Hartmann, N. Lieser, Ch. Schultheiss, R. Seebock, and W. Steudtner, Nucl. Instrum. Methods 205, 173 (1983).

[3] G. F. Kirkman and M. A. Gundersen, Appl. Phys. Lett. 49, 494 (1986).

[4] H. Riege and E. Boggasch, IEEE Trans. Plasma Sci. PS-17, 775 (1989).

[5] P. Choi, H. H. Chuaqui, M. Favre, and E. S. Wyndham, IEEE Trans. Plasma Sci. PS-15, 428 (1987).
[6] M. Hobel, J. Geerk, G. Linker, and C. Schultheiss, Appl. Phys. Lett. 56, 973 (1990).

[7] E. Boggasch and M. J. Rhee, Appl. Phys. Lett. 56, 1746 (1990).

[8] K. K. Jain, E. Boggasch, M. Reiser, and M. J. Rhee, Phys. Fluids B 2, 2487 (1990).

[10] K. Frank, Physics and Applications of Pseudosparks, edited by M. A. Gundersen and G. Schaefer, (Plenum, New York, 1990), p. 15.

[9] P. R. Bevington, Data Reduction and Error Analysis for the Physical Sciences, (McGraw-Hill, New york, 1969), p. 180 .

[11] M. J. Rhee and R. F. Schneider, Part. Accel. 20, 133 (1986). 